\title{
Technologists' Forum: Roundtable Discussion of Energy Dispersive Spectroscopy
}

\section{E. Ann Ellis}

\author{
Microscopy and Imaging Center, Texas A\&M University, College Station, TX 77843-2257
}

Over the years energy dispersive spectroscopy (EDS) has proven to be a useful research tool and now lends itself to both materials and biological specimens. For some specimens, EDS presents some special problems in specimen preparation and demands that we properly define and understand what we can and cannot accomplish with this methodology. Analysis of bulk materials samples can be quite straightforward or not so straightforward. Elemental analysis of biological materials is often challenging as a result of loss of elements during specimen preparation in the aqueous solvents used in both fixation, dehydration, embedding and or coating to stabilize the samples before exposure to the electron beam.

Prior to initiating EDS analysis the sample should be evaluated in terms of what is expected. Are you doing survey work or are you looking for certain elements? First, does the element have a minimal detectable concentration in the specimen? Is it practical to do EDS when a simpler, wet chemistry protocol might provide just as good or better elemental analysis? It may not be possible to detect an element below a certain threshold. Are there other elements which might interfere with detection of a particular element peak? Osmium tetroxide is often used to fix biological samples and reduces charging; however, osmium can overlap or obscure other elements. A solution to this problem can be the use of $1-2 \%(\mathrm{wt} / \mathrm{vol}) \mathrm{p}$-phenylenediamine in the dehydration alcohols [1].

Specimen preparation involves $90 \%$ or more of the work done by electron microscopists. If the preparation is done well, the work goes smoothly and consistently. How will the specimen be prepared in order to insure an accurate analysis? Both materials and biological samples can be damaged or contaminated by improper handling. Elements like sodium, potassium and chlorine from sweaty hands can come from handling the specimen with dirty hands or dirty tools. Some surface elements can be removed or disturbed by washing with various solvents including water. Will it be necessary to coat the specimen with carbon or some other coating to minimize charging and carbon may or may not be the best coating for a particular specimen. Are there possible sources of peak overlaps in your specimen? Could the coating you used cause peak overlaps or other problems? Is the specimen properly grounded? Is the specimen properly tilted toward the detector? Do you use a standard tilt angle toward the detector or do you work with the tilt angle until you think it is optimised?

Is the bulk specimen dry or how will you insure that it is dry? Charging can interfere with both imaging as well as EDS analysis. You can improve the speed of turn around by pumping bulk specimens down overnight in a high vacuum evaporator.

Do you know what chemistry was involved in producing your sample that might account for some of the results you are seeing? Are you familiar with the morphology of the specimen? Have you considered backscatter imaging to learn more about the location of high atomic number versus low atomic number elements in the specimen? Is the electron microscope properly aligned? Is your EDS system properly calibrated? What accelerating voltage will you use for both imaging and EDS analysis? Do you have an algorithm for standard analysis by EDS? 
Have you talked to an experienced EDS analyst about your problem? If this particular specimen is unusual, have you consulted the literature about the specimen preparation or any other related details? Do you keep up with what is going on with the MSA Listserver or similar resources? Do you read Microscopy Today or other similar, free publications from time to time? There are often interesting topics that might give you some insight into your particular problem.

The series of questions presented here are just some of the questions that you may or may not consider when you are trying to improve on a particular analysis. Standard protocols are important; but sometimes you need to think outside the box. Hopefully, participation in this meeting and the "Technologists' Forum Round Table Discussion on Basic EDS" will have a positive impact on your and your colleagues' work.

\section{References}

1. EA Ellis Microscopy Today 18 (2010), 48. 